\title{
Research Paper: Effects of Extracorporeal Shock Wave Therapy on Muscle Spasticity in Post-Stroke Pa- tients: An Ultrasonography and Clinical-Base Study
}

\author{
Maryam Rastgoo ${ }^{1}$, Hadi Sarafraz ${ }^{2}$, Hamidreza Najari $^{3}$, Mohammad Reza Hadian $^{4}$, Bijan Forough ${ }^{5}$ Asghar Rezasoltani $^{6 *}$ \\ 1. Department of Physical Therapy, School of Rehabilitation Sciences, Shahid Beheshti University of Medical Sciences, Tehran, Iran. \\ 2. Department of Neurology, Shahid Mohammadi Hospital, Hormozgan University of Medical Sciences, Bandarabbas,Iran. \\ 3. Department of Internal Midicine School of Medicine, Qazvin University of Medical Sciences, Qazvin, Iran. \\ 4. Department of Physiotherapy, School of Rehabilitation, Tehran University of Medical Sciences, Tehran, Iran. \\ 5. Department of Physical and Rehabilitation Medicine, School of Medicine, Iran University of Medical Sciences, Tehran, Iran. \\ 6. Physiotherapy Research Centre, School of Rehabilitation, Shahid Beheshti University of Medical Sciences, Tehran, Iran.
}

Cttation: Rastgoo M, Sarafraz H, Najari H, Hadian MR, Forough B, Rezasoltani A. Effects of Extracorporeal Shock Wave Therapy on Muscle Spasticity in Post-Stroke Patients: An Ultrasonography and Clinical-Base Study. Physical Treatments. 2016; 6(3):169-178. https://doi.org/10.18869/ NRIP.PTJ.6.3.169

: https://doi.org/10.18869/NRIP.PTJ.6.3.169

Article info:

Received: 11 Jun, 2016

Accepted: 31 Aug, 2016

Keywords:

Stroke, Spasticity, Extracorporeal shock wave therapy, Gait, Muscle architecture

\section{A B S T R A C T}

Purpose: To investigate the effect of single session Extracorporeal Shock Wave Therapy (ESWT) over the ankle plantar flexor muscles on the spasticity, muscle architecture, and gait in chronic stroke patients.

Methods: This quasi-experimental, single group study had a repeated measures design. A total of 17 post-stroke patients were selected by convenience sampling method, and received 2000 shots of ESWT on the ankle plantar flexor muscles of the affected side in one session. Outcome measures consisted of Modified Modified Ashworth Scale (MMAS), bilateral leg circumference, and muscle architecture parameters (pennation angle, fascicle length, and muscle thickness) of the medial head of the gastrocnemius muscle, timed up and go (TUG) test, self-reported visual analog scale (VAS) of spasticity, and ankle passive Range of Motion (pROM). All outcomes were measured at 2 times: before the intervention and 30 minutes after the intervention. The muscle architecture parameters and leg circumference were also measured immediately after the intervention. Statistical analysis was done using SPSS 18.0. Repeated measures analysis of variance (ANOVA) was performed to evaluate the effects of intervention in time.

Results: Wilcoxon test revealed significant improvement in MMAS. Significant improvement in VAS, pROM, and TUG test results is also noted by means of the paired $t$ test. ANOVA showed significant time $\times$ side interaction for the pennation angle and leg circumference. Significant effect of time was also shown by ANOVA for leg circumference. Post hoc analyses showed a significant difference for the leg circumference before the intervention and 30 minutes after it. ANOVA showed no significant change in fascicle length and muscle thickness. Spearman's rho revealed significant positive correlation between the changes of the ankle pROM and improvement of the fascicle length.

Conclusion: According to the results single session of ESWT over the spastic plantar flexors muscle can improve clinical measures of muscle spasticity and motor function. More studies are needed to clarify the underlying mechanisms for the antispastic effect of ESWT.

\section{* Corresponding Author:}

Asghar Rezasoltani, PhD

Address: Physiotherapy Research Centre, School of Rehabilitation, Shahid Beheshti University of Medical Sciences, Tehran, Iran

Phone: +98 (912) 2975807

E-mail: a_rezasoltani@sbmu.ac.it 


\section{Introduction}

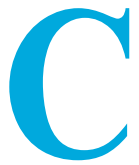

erebrovascular accident or stroke is the most common cause of the adult neurological disability around the world [1]. Following to the stroke, motor impairments such as spasticity and paresis may occur. Spasticity is defined as a velocity-dependent increase in tonic stretch reflexes (muscle tone) with exaggerated tendon jerk, resulting from hyperexcitability of the stretch reflex [2]. The prevalence of spasticity one year after the first ever stroke is reported to be $20 \%-30 \%$ of all stroke victims and less than half of those with paresis [3]. Following the stroke, the morphologic changes in architecture of spastic muscles can be evaluated by musculoskeletal ultrasonography. Fascicular changes of spastic muscles compared to normal ones were reported in previous studies $[4,5]$. Untreated spasticity hinders normal motor function and reduces patients' independence in daily livings activities. Therefore, treatment of spasticity has been one of the health care concerns since many years ago. Different invasive and noninvasive treatments have been used for spasticity treatment.

Extracorporeal Shock Wave Therapy (ESWT) is a physical modality, which has been used for various musculoskeletal problems. ESWT is defined as a series of single sonic pulse characterized by spike pressure (100 $\mathrm{MPa})$, rapid rise $(<10 \mathrm{~ns})$, and brief duration $(10 \mu \mathrm{s})$, applied by an appropriate generator to a specific target area with the range of $0.003-0.89 \mathrm{~mJ} / \mathrm{mm}^{2}$ energy density [6]. Previous studies evaluated the effect of ESWT on spasticity in different neurological conditions such as cerebral palsy [7-10], stroke [11-16], and multiple sclerosis [17]. In previous studies with ESWT to treat post-stroke plantarflexor spasticity, the effects were not evaluated in terms of clinical, functional, and muscle architecture measures altogether. Also, the correlation between muscle spasticity improvement and muscle architecture changes following ESWT has been completely overlooked. The present study aimed to investigate the effect of single session of ESWT over the spastic plantar flexor muscles on muscle spasticity, gait, and muscle architecture in stroke population.

\section{Materials and Methods}

\section{Study design}

This quasi-experimental, single group study had a repeated measures design. The study design was approved by the Ethics Committee of Shahid Beheshti University of Medical Sciences. The study was conducted from June to August 2015.

\section{Study participants}

Out of 31 participated patients, 17 were selected by convenience sampling method. The inclusion criteria were as follows: 1 ) aged $\geq 18$ years; 2 ) time past since onset of stroke $\geq 6$ months; 3 ) ankle plantar flexors spasticity (at least MMAS grade 1); and 4) ability to walk independently (with or without walking aids). The exclusion criteria were as follows: 1) past history of surgical procedure in the lower extremity, 2) MMAS grade 4 or more, 3) use of anti-spastic drugs, and 4) local injection of botulinum toxin type $\mathrm{A}$ in the past 3 months.

Subjects who met the inclusion criteria and did not have exclusion criteria were assigned to the study. Poststroke patients were recruited from rehabilitation center of Firoozgar Hospital affiliated to Iran University of Medical Sciences and Stroke Rehabilitation Clinic affiliated to School of Rehabilitation Science of Shahid Beheshti University of Medical Sciences.

\section{Study procedure}

After description of the study procedure to the patients, the informed consent was obtained from them. Demographic features of the patients consisting of age, height, weight, and time past since the stroke onset were taken. After measuring the pre-intervention outcomes, all patients received single session of ESWT. Post-intervention outcome measurements (leg circumference and ultrasonographic parameters) were done at two times: one, immediately after the intervention and the other 30 minutes later.

As any active or passive movement of the ankle may affect the morphologic features of the muscle architecture, patients were asked to keep on the primary prone position till the other ultrasonographic measurements (30 minutes post intervention). Other outcomes were measured after the ultrasonographic measurements. All outcomes were measured by a well-trained physiotherapist and intervention was done by another physiotherapist. In order to evaluate the intra-rater reliability of the ultrasonography measurments, 2 sets of imaging with one hour interval were taken from 7 patients in a separate session.

\section{Outcome measures}

Primary outcome measures were Modified Modified Ashworth Scale (MMAS) to assess ankle plantar flexor muscles spasticity and ultrasonographic evaluation of 
the medial head of the gastrocnemius muscle to assess muscle architecture. Secondary outcome measures were timed up and go (TUG) test for gait evaluation, ankle passive dorsiflexion range of motion (pROM), leg circumference, and self-reported evaluation of spasticity using visual analog scale (VAS).

\section{Spasticity evaluation}

The ankle plantar flexor muscles spasticity was assessed by MMAS. It is an ordinal level measure of spasticity, grading its intensity from 0 to 4 based on the resistance to a quick and passive movement. Based on MMAS score, 0 means no increase in muscle tone and 4 means the affected part is rigid in flexion or extension. It has been proved that MMAS is a reliable tool for measuring ankle plantar flexors post-stroke spasticity. For evaluating the ankle plantar flexor muscles spasticity, the patient was in supine position, with his or her lower limbs in extension. The physiotherapist stabilizes the ankle with her left hand and moved the ankle from maximal plantar flexion to dorsiflexion with her right hand [19].

\section{Ultrasonographic evaluation of muscle architecture}

In order to evaluate the architecture of the spastic plantar flexor muscles, ultrasonographic imaging from the medial head of the gastrocnemius muscle was performed bilaterally. Reliability of ultrasonographic measurements of this muscle in post-stroke patients was shown in previous study [20]. In this study, intra-rater reliability of the examiner was also evaluated.

In the present study, real-time B-mode ultrasonography (Medison X8, Medison Co. South Korea) with $8.5 \mathrm{MHz}$ linear array probe was used. During ultrasonography, the patient was asked to lie in the prone position while his or her feet were suspended over the end of the bed. The hip joint had no rotation, the knee joint was kept in full extension, and the ankle was in its resting position as the ultrasonography was being done. The leg length which was the distance between the popliteal crease and the center of the lateral malleolus was measured. The border between the medial and lateral head of the gastrocnemius muscle was determined by moving the linear probe horizontally in a line corresponding to the proximal third of the leg length.

The distance between this point and the medial border of the leg was marked on the skin. This skin mark was kept throughout the study. The medial gastrocnemius image was obtained by putting the center of the probe on this point with minimal pressure to avoid muscle fiber compression. The distance between the superficial and

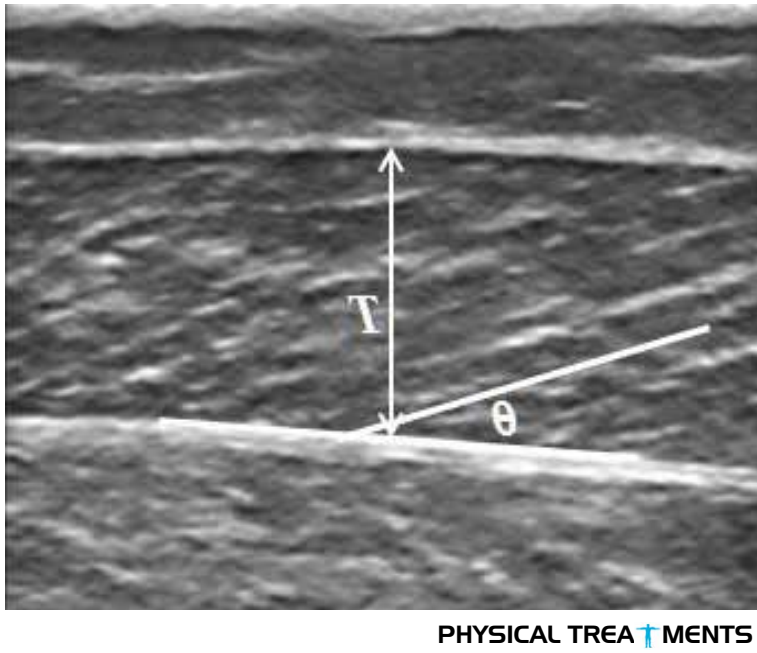

Figure 1. Ultrasonographic image of the medial head of gastrocnemius muscle at rest. T, and correspond to the muscle thickness and pennation angle respectively.

deep aponeurosis was considered as the muscle thickness and the angle between the muscle fascicles and deep aponeurosis was regarded as the pennation angle (Figure 1). As the entire length of the muscle fascicle was not seen in the image, the fascicle length was measured by using the following formula:

\section{Fascicle Length $=\sin \theta^{-1} \times$ Muscle Thickness}

where $\theta$ is the pennation angle. All images were taken by the same examiner. Three images from the affected and unaffected side were taken at each assessment time (pre-intervention, immediately, and 30 minutes postintervention) and the average value was calculated at each assessment time. In order not to interact with the effect of the intervention on the muscle, the patients were asked to stay in the same position and not to move their leg during the 3 levels of assessments [4].

\section{Intra-rater reliability for the ultrasonographic} measurements

Seven patients were randomly chosen for reliability study. The intra-rater reliability of the pennation angle, muscle thickness, and fascicle length measurements was done in a separate session from the intervention session. Muscle ultrasonography was performed at two times with one hour interval. At each time, 3 images were taken from the medial head of the gastrocnemius muscle of the affected side by the same examiner. The average value was considered for statistical analysis [20]. During these tests the leg position and the measurements were the same as the ultrasonographic measurements of the study which was described before. Patients remained in 
prone position and did not move the leg throughout the reliability study.

\section{Leg circumference}

After measuring the leg length, the diameter of the leg at the point corresponding to the first third of the leg length was measured by a metric tape. This measurement was done for both the affected and the unaffected legs.

\section{Gait evaluation}

Timed up and go test (TUG) was used for gait evaluation. TUG is a simple and quick functional mobility test. It includes a series of motor tasks that require balanced control, muscle strength, and coordination [21]. Excellent reliability for this test was reported in chronic post-stroke patients [22]. For performing the test, the patient is instructed to stand up from a chair with an armchair, walk $3 \mathrm{~m}$ forward, turn, walk back, and sit down on the same chair. The time taken to complete the test is recorded [21, 22].

\section{Ankle passive range of motion (pROM)}

Standard method for ankle passive range of motion (pROM) measurements was used by means of a manual goniometer [23]. In order to measure the passive ROM (pROM), the patient lies in supine position while his or her knee is kept in extension and hip with no rotation. The examiner stands by the patients measuring side and move the foot to the maximum dorsiflexion while the knee is kept in extension. The center of the goniometer is placed to the center of the lateral malleolus. The fixed arm of the goniometer is aligned to the shaft of the fibula and the moving arm is aligned to the first metatarsal. The positive and negative values from the position of $90^{\circ}$ (zero position) indicate the ankle dorsiflexion and plantarflexion, respectively.

\section{Self-reported evaluation of spasticity using visual analog Scale}

Visual Analog Scale (VAS) is used to measure the severity of the self-reported spasticity. The validity and reliability of VAS for spasticity measurement have been reported in previous studies. The patient is instructed to rate his or her muscle spasticity during walking. The VAS used in this study was a straight line with 10 scores which the anchor points on the left side of the line corresponded to no difficulty related to muscle spasticity (score 0 ) and the end point on the right side of the line to unbearable difficulty related to muscle spasticity (score 10) [24].

\section{Intervention}

An ESWT device (BTL Industries Ltd, United Kingdom) with a $15 \mathrm{~mm}^{2}$ diameter of the probe head was used for shock wave therapy. The patients received single session of 2000 shots of ESWT over the spastic plantar flexor muscles of the affected side. The shots were distributed to the bulks of the medial head and lateral head of the gastrocnemius and the soleus muscles (600 shots on each one) and the Achilles tendon (200 shots) [17]. The applied frequency was $5 \mathrm{~Hz}$, with a pressure of 1.5 Bars. During the intervention, the patients lay in prone position while their feet were suspended over the end of the bed.

\section{Statistical analysis}

All statistical analysis was done using SPSS 18.0. The significant level was defined as $\mathrm{P} \leq 0.05$. Descriptive statistics were done and the value of the mean and standard deviation (SD) for the parameters were reported. As the criterion for normality (Shapiro-Wilk test) was met, repeated measures analysis of variance (ANOVA) was performed to evaluate the effects of intervention (affected vs. unaffected lower extremity) at three time points (pre-intervention, immediately, and 30 minutes postintervention) on pennation angle, fascicle length, muscle thickness and leg circumference. Effect sizes for F-ratios were reported as eta-squared $\left(\eta^{2}\right)$. Post hoc analysis was performed with Bonferroni correction.

For the outcomes which were done at pre-intervention and 30 minutes post-intervention, such as TUG, pROM, and VAS, paired t test were done. For MMAS, median and quartiles range were calculated and as it is an ordinal level measurement, the Wilcoxon signed-rank test was conducted. In order to compare the 2 side (affected vs. unaffected sides) measurements, the independent sample $\mathrm{t}$ test was done.

One-way random effects model (people effect random, single rater) intra-class correlation coefficient (ICC) statistics [25] was used to assess the intra-rater reliability of the ultrasonographic measurements. The ICC values were classified as low (0.00-0.039), moderate (0.40-0.59), moderately high (0.60-0.80), and high (0.80-1.00) [26]. Standard error of measurement (SEM) and minimal detectable change (MDC) were also calculated for the ultrasonographic measurements with the following formula [27]:

$$
S E M=S D \times \sqrt{1-I C C} \text { and } M D C=\sqrt{ } 2 \times 1.96 \times S E M
$$

Spearman's rho correlation test was applied to detect the relationship between pROM improvement and spas- 
ticity reduction (MMAS) with the change in ultrasonographic measurements of the medial head of the gastrocnemius muscle of the affected side.

\section{Results}

Out of eligible participants, 17 were enrolled in the study. The characteristics of the patients are presented in Table 1 .

\section{Modified ashworth scale}

Ankle plantar flexor muscles spasticity was evaluated by MMAS at two times: before the intervention and $30 \mathrm{~min}-$ utes after the intervention. The median (Mdn) and interquartiles range (IQR) of the MMAS improved 30 minutes after the intervention ( $\mathrm{Mdn}=1, \mathrm{IQR}=1-2)$ in comparison to the pre-intervention ( $\mathrm{Mdn}=2, \mathrm{IQR}=2-2)$. The Wilcoxon signed-rank test showed a significant decrease in MMAS score before and after the intervention $(\mathrm{P}<0.01)$.

Intra-rater reliability for the ultrasonographic measurements

Intra-rater reliability for the fascicle length, pennation angle, and muscle thickness was high. SEM and MDC for the fascicle length, pennation angle, and muscle thickness were also calculated. These values are presented in Table 2.

Ultrasonographic measurements of the muscle architecture

Descriptive data of the ultrasonographic measurements of the medial head of the gastrocnemius muscle for both the affected and unaffected side are provided in Table 3. These data show the steady decrease of the pennation angle as well as the muscle thickness after the intervention on the affected side. Meanwhile the fascicle length of the affected side increases and continues 30 minutes post-intervention.

Repeated measures ANOVA showed no significant effect of time for any muscle architecture parameters. Significant interaction of time $\times$ side was shown for the pennation angle $\left[\mathrm{F}(2,64)=4.29, \mathrm{P}=0.02, \eta^{2}=0.12\right]$, whilst this interaction was neither significant for the muscle thickness nor for the fascicle length. The main effect of side is not significant for any muscle architecture parameters.

Independent sample $t$ test showed no significant difference between the affected and unaffected side for the fascicular pennation angle, fascicle length, muscle thickness and leg

Table 1. Characteristics of the participants.

\begin{tabular}{cc}
\hline Variables & \\
\hline Age(y), mean(SD) & $52.1(11.1)$ \\
\hline Gender(male/female) & 14.3 \\
Affected side(right/left) & 4.13 \\
\hline Time since the stroke(months), mean(SD) & $17.1(6.9)$ \\
\hline BMI(kg/m²), mean(SD) & $24.6(3.4)$ \\
\hline Type of stroke, ischemic/hemorrhagic & 1215 \\
\hline
\end{tabular}

Abbreviations: SD: Standard Division; BMI: Body Mass Index

PHYSICAL TREA TMENTS

Table 2. The values for the intra-rater reliability of the ultrasonographic measurements of the medial head of the gastrocnemius muscle of the affected side in 7 patients.

\begin{tabular}{|c|c|c|c|c|}
\hline & ICC (95\%CI) & $\mathbf{P}$ & SEM & MDC \\
\hline Pennation angle (degree) & $0.93(0.6-0.98)$ & 0.02 & 0.56 & 1.53 \\
\hline Muscle thickness (mm) & $0.99(0.95-0.99)$ & $<0.001$ & 0.54 & 1.48 \\
\hline Fascicle length (mm) & $0.99(0.86-0.99)$ & $<0.001$ & 1.8 & 4.9 \\
\hline
\end{tabular}

PHYSICAL TREA \MENTS

Abbreviations: ICC: Interclass Correlation Confidence; CI: Confidence Interval; SEM: Standard Error of Measurement; MDC: Minimal Detectable Change 
Table 3. Values of the ultrasonographic parameters (pennation angle, muscle thickness and fascicle length) of the medial head of the gastrocnemius muscle and leg circumference in time (different times of assessment) for both the affected and unaffected sides.

\begin{tabular}{ccccc}
\hline & & $\begin{array}{c}\text { Affected Side } \\
\text { Mean (SD) }\end{array}$ & $\begin{array}{c}\text { Unaffected Side } \\
\text { Mean (SD) }\end{array}$ & $\begin{array}{c}\text { Affected vs. Unaffected Side } \\
\text { P }\end{array}$ \\
\hline \multirow{2}{*}{$\begin{array}{c}\text { Pennation angle } \\
\text { (degree) }\end{array}$} & Before the intervention & $22.6(2.9)$ & $21.0(3.5)$ & 0.16 \\
& Immediately after the intervention & $21.9(2.9)$ & $20.8(3.4)$ & 0.3 \\
Muscle thickness & 30 Minutes after the intervention & $21.5(2.9)$ & $21.4(3.3)$ & 0.9 \\
(mm) & Before the intervention & $14.2(3.2)$ & $14.9(4)$ & 0.57 \\
Immediately after the intervention & $14.1(3.1)$ & $14.7(4)$ & 0.59 \\
Fascicle length & 30 Minutes after the intervention & $13.8(3)$ & $14.8(3.7)$ & 0.38 \\
(mm) & Before the intervention & $37.6(9)$ & $42.3(9.6)$ & 0.15 \\
Immediately after the intervention & $38.2(7.9)$ & $42.8(10.2)$ & 0.19 \\
Leg circumference & 30 Minutes after the intervention & $38.5(9.5)$ & $41.1(8.4)$ & 0.4 \\
(cm) & Before the intervention & $35.5(3.7)$ & $36.3(3.5)$ & 0.53 \\
\hline
\end{tabular}

Abbreviation: SD: Standard Division.

PHYSICAL TREA \MENTS

circumference at any time of the assessment (before, immediately after, and 30 minutes after the intervention).

\section{Leg circumference}

Descriptive data of the bilateral leg circumference are presented in Table 3. Leg circumference of the affected side decreased after the intervention. Repeated measures ANOVA showed significant effect of time on the leg circumference $\left[\mathrm{F}(2,64)=4.87, \mathrm{P}=0.01, \eta^{2}=0.13\right]$. Post hoc analyses showed a significant difference between before the intervention and 30 minutes after it (leg circumference mean differences $=0.18, \mathrm{P}=0.03$ ). Although the main effect of side was not significant, interaction of time and side for the leg circumference was significant $\left[F(2,64)=5.15, \mathrm{P}<0.01, \eta^{2}=0.14\right]$.

\section{TUG test}

As it is shown in Table 4, the time to complete the TUG test decreased after the intervention in comparison to before the intervention. Comparing the means by paired $\mathrm{t}$ test, significant changes for the TUG test, 30 minutes post-intervention in comparison to pre-intervention was shown $(\mathrm{P}<0.001)$.

\section{VAS for muscle spasticity}

VAS score decreased after the intervention (Table 4). Paired t test revealed significant changes for the VAS, 30 minutes after the intervention in comparison to it before the intervention $(\mathrm{P}<0.001)$.

Table 4. Values of the outcome measures before the intervention and 30 minutes after the intervention.

\begin{tabular}{ccccc}
\hline & $\begin{array}{c}\text { Before the Intervention } \\
\text { Mean (SD) }\end{array}$ & $\begin{array}{c}\text { 30 Minutes After the Intervention } \\
\text { Mean (SD) }\end{array}$ & t & P \\
\hline TUG test (seconds) & $34.8(22.71)$ & $31.18(21.72)$ & 4.4 & $<0.001$ \\
VAS & $5.52(1.66)$ & $4.53(0.73)$ & 4.4 & $<0.001$ \\
PROM (degree) & $-7(6.13)$ & $-3.05(4.77)$ & -3.96 & 0.001 \\
\hline
\end{tabular}

PHYSICAL TREA T MENTS

Abbreviations: SD: Standard Division; TUG: Timed Up and GO; VAS: Visual Analog Scale; MMAS: Modified Modified Ashworth Scale; pROM: Passive Range of Motion 


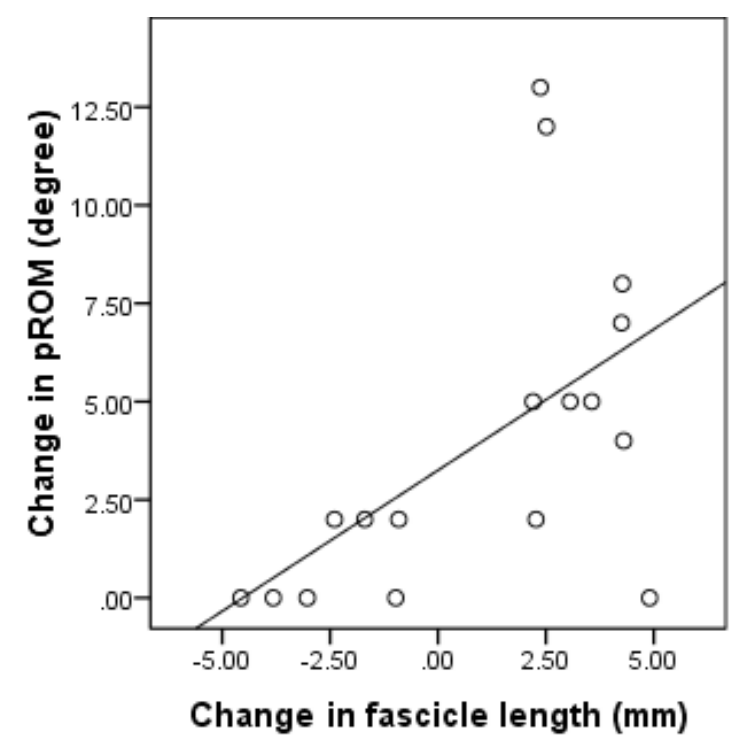

PHYSICAL TREA \MENTS

Figure 2. Correlation between the changes in fascicle length and pROM (Passive Range of Motion) 30 minutes post-intervention in comparison to pre-intervention status.

\section{Ankle passive ROM}

Ankle passive dorsi flexion ROM improved 30 minutes after the intervention (Table 4). Significant changes for pROM 30 minutes post-intervention in comparison to pre-intervention was revealed by means of the paired $t$ test $(\mathrm{P}=0.001)$.

\section{Correlation}

Spearman's rho test and linear regression was used for detecting the correlations. The analysis showed significant negative correlation between the spasticity reduction and fascicle length improvement $\left(\mathrm{r}=-0.6, \mathrm{R}^{2}=0.2, \mathrm{P}=0.02\right)$. The correlation of muscle thickness and pennation angle improvement with spasticity reduction, $\left(\mathrm{r}=-0.18, \mathrm{R}^{2}=0.05\right.$, $\mathrm{P}=0.48),\left(\mathrm{r}=0.24, \mathrm{R}^{2}=0.07, \mathrm{P}=0.34\right)$ respectively, were shown to be insignificant by Spearman's rho test.

Spearman's rho revealed significant positive correlation between the changes of the ankle $\mathrm{pROM}$ and the improvement of the fascicle length $\left(\mathrm{r}=0.55, \mathrm{R}^{2}=0.3, \mathrm{P}=0.02\right)$ (Figure 2). The correlation between the changes of the pROM and neither pennation angle nor the muscle thickness were significant $(\mathrm{r}=-0.2, \mathrm{P}=0.4),(\mathrm{r}=0.41, \mathrm{P}=0.08)$.

\section{Discussion}

The study results showed that one session of ESWT on the ankle plantar flexor muscles improve muscle spasticity and gait function. The positive effect of ESWT on spasticity reduction in different upper motor neuron diseases such as cerebral palsy [7-10], stroke [11-16] and multiple sclerosis [17] has been shown in previous studies. Positive effect of ESWT on the feet plantar surface contact has been also shown in studies which applied ESWT over the spastic ankle plantar flexor muscles [7,9].

By evaluating the muscle architectural properties of medial head of the gastrocnemius muscle by ultrasonopgraphy, Gao et al. revealed the changes at the fascicular level. These changes consisted of the smaller pennation angle, shortened fascicle length, and thinner muscle thickness of the spastic muscle of stroke patients in comparison to the control healthy group [4]. In the present study, muscle architecture measurements of the medial head of the gastrocnemius were done bilaterally. Our results showed that in comparison to the unaffected side, the mean data of the fascicle length and muscle thickness were lower and the pennation angle was higher in the affected side. However, no significant difference in the muscle architecture parameters was detected between the affected and unaffected sides with regard to these measurements. It should be mentioned that the discrepancy between the result of Gao et al. study and ours may be due to their different control groups.

In Gao et al. study, the control group was healthy people whilst in our study the unaffected leg was considered as the control group. It is believed that the unaffected side of the hemiplegic patients cannot be considered as a healthy side [28]. Another reason for this discrepancy may be due to the differences in the time past since onset of the stroke in two studies. Gao et al. included stroke patients that at least 1 year has passed their stroke, meanwhile in the present study 4 participants (23\%) experienced their stroke 6 to 12 before. Therefore, it seems that many factors such as the duration after the stroke onset and the degree of spasticity can affect the spastic muscle architecture.

Apparently with more time past the stroke, the changes in muscle architecture become more similar to the changes of an atrophic muscle [16]. It was revealed that the gastrocnemius muscle is the most susceptible muscle of the lower extremity to non-use which was detected by the changes in the muscle architecture [27]. It also seems that the trend of changes in the upper and lower extremity muscle architecture of the affected side following stroke is not similar [4, 5]. More studies in different phases after stoke and for both the upper and lower extremity are necessary to investigate the muscle architecture changes and its effects on spasticity and muscle function.

In the present study, the ultrasonographic measurements changed after the intervention; however, these changes neither reach the statistically significant level, 
nor being meaningful according to MDC. Insignificant difference between the affected and unaffected side may indicate the minority of morphological changes in the spastic muscle architecture in this group of patients. Therefore, if the changes in the muscle architecture were more, the effect of the intervention on it would be significant. The result showed that the changes in the muscle architecture continue steadily [30] minutes after the intervention. It was claimed that the effect of ESWT on spasticity is long lasting; more changes in muscle architecture may be detected if we did the ultrasonographic measurement one day or one week after the intervention.

In the present study, significant negative correlation was observed between fascicle length improvement and MMAS reduction. The correlation between spasticity score and muscle architecture of the spastic medial gastrocnemius muscle was also shown in previous study 30. This finding may imply the muscle role on spasticity at the fascicular level. Considering the reduction of the fascicule length in post-stroke spastic muscle, more fascicular tension is produced during passive stretch, which may play a part in non-reflex element of spasticity. Therefore, despite insignificant change of the fascicule length, it has some effect on spasticity reduction. So this may suggest that the muscle fascicular changes as an underlying mechanism for spasticity.

In this study, the improvement of spasticity was evaluated by using clinical measures such as MMAS, VAS, leg circumference, and passive ROM as well as muscular ultrasonographic measurements. Although different mechanisms for ESWT effect on musculoskeletal problems have been known, the exact mechanism of ESWT in muscle spasticity improvement is not fully understood yet. It was believed that ESWT can reduce motor neuronal excitability and cause peripheral nerve injury by the mechanical vibratory stimulation induced on the tissues beneath. This theory was ruled out, since no change in electrophysiologic measures of the motor neuron excitability was reported following ESWT for spastic plantar flexor muscles [14, 16, 17]. The direct effect of ESWT on the connective tissue stiffness and muscle fibrosis is proposed by other authors as the main mechanism for its effects on spasticity $[7,16,17]$. In our study, despite improvement in the ankle ROM and spasticity, ESWT had minor effect on muscle architecture at the fascicular level; therefore reducing spasticity following ESWT through its effect on fibrosis and rheological properties of chronic spastic muscle is also proposed as the antispastic mechanisms of ESWT in this study. Possible effect of ESWT on non-reflex elements of spasticity such as improving the stiffness of the connective tissue by di- rectly acting on fibrosis can be evaluated by elastosonography in future studies. Other known mechanisms for ESWT effect on musculoskeletal diseases such as Nitric Oxide (NO) formation should be evaluated as antispastic mechanism in future studies.

The significant effect of ESWT on gait was shown in this study. Previous study by Amelo and Manganotti also showed that one session ESWT on the spastic plantar flexor muscles was effective on the reduction of spasticity, improvement of the postural attitude, body stability and the whole plantar surface area of the foot [8]. Positive effects of four sessions of ESWT on spastic plantar flexor muscles on these parameters were also shown in a study by Gonkova et al. [9]. Spasticity of the plantar flexor muscles and the equionovarus deformity is considered as causes of gait problems in stroke population. Therefore, the improvement in plantar flexor muscles spasticity and ankle ROM can account for the detected improvement in gait.

This study has some limitations, too. Firstly, participants were a few and sampling was of non-randomized (convenience) model; Secondly, there was no control group and all patients received the same intervention; Thirdly, as any movement of the foot may disturb the absolute effect of the intervention on the muscle, some measures were not done immediately after the intervention; Fourthly, in order to evaluate the effect of ESWT on the morphological features of the plantar flexor muscles, it is better to do the muscular ultrasonography for the lateral head of the gastrocnemius as well as the soleus muscles and the Achilles tendon; Fifthly, since it is thought that the antispastic effect of ESWT is through the improvement in the connective tissue stiffness by its direct effect on fibrosis, this effect can be evaluated by means of muscular elastosonography in future studies; Sixthly, as the long-term effect of ESWT on spasticity was shown in previous studies, the long-term effect of ESWT on the plantar flexor muscles architecture could be evaluated; seventhly, as ESWT is usually paired with other interventions in clinical practice, further research is needed to investigate its potential synergic effect by pairing ESWT with exercise or stretching program.

One session ESWT on spastic ankle plantar flexor muscles in stroke patients reduced the spasticity and improved their gait. Further research is needed to understand the exact antispastic mechanism of ESWT.

\section{Acknowledgments}

This study was financially supported by Physiotherapy Research Centre, School of Rehabilitation Sciences, 
Shahid Beheshti University of Medical Sciences. Special thanks to the Department of Physical Medicine and Rehabilitation of Firoozgar University Hospital for their kind cooperation.

\section{Conflict of Interest}

The authors report no conflicts of interest.

\section{References}

[1] Lavados PM, Hennis AJ, Fernandes JG, Medina MT, Legetic B, Hoppe A, et al. Stroke epidemiology, prevention, and management strategies at a regional level: Latin America and the Caribbean. The Lancet Neurology. 2007; 6(4):362-72. doi: 10.1016/s1474-4422(07)70003-0

[2] Lance JW. The control of muscle tone, reflexes, and movement: Robert Wartenbeg Lecture. Neurology. 1980; 30(12):1303. doi: 10.1212/wnl.30.12.1303

[3] Sommerfeld DK, Gripenstedt U, Welmer AK. Spasticity after stroke. American Journal of Physical Medicine \& Rehabilitation. 2012; 91(9):814-20. doi: 10.1097/phm.0b013e31825f13a3

[4] Gao F, Grant TH, Roth EJ, Zhang LQ. Changes in passive mechanical properties of the gastrocnemius muscle at the muscle fascicle and joint levels in stroke survivors. Archives of Physical Medicine and Rehabilitation. 2009; 90(5):819-26. doi: 10.1016/j.apmr.2008.11.004

[5] Li L, Tong KY, Hu X. The effect of poststroke impairments on brachialis muscle architecture as measured by ultrasound. Archives of Physical Medicine and Rehabilitation. 2007; 88(2):243-50. doi: 10.1016/j.apmr.2006.11.013

[6] Bisset L. A systematic review and meta-analysis of clinical trials on physical interventions for lateral epicondylalgia, commentary. British Journal of Sports Medicine. 2005; 39(7):411-22. doi: 10.1136/bjsm.2004.016170

[7] Amelio E, Manganotti P. Effect of shock wave stimulation on hypertonic plantar flexor muscles in patients with cerebral palsy: A placebo-controlled study. Journal of Rehabilitation Medicine. 2010; 42(4):339-43. doi: 10.2340/16501977-0522

[8] AmAmelio E, Manganotti P. Effect of shock-wave therapy on spastic equinus foot in patients affected by cerebral palsy. Journal of Neurology. 2006; 253(2):152.

[9] Gonkova MI, Ilieva EM, Ferriero G, Chavdarov I. Effect of radial shock wave therapy on muscle spasticity in children with cerebral palsy. International Journal of Rehabilitation Research. 2013; 36(3):284-90. doi: 10.1097/ mrr.0b013e328360e51d

[10] Wang T, Du L, Shan L, Dong H, Feng J, Kiessling MC, et al. A prospective case-control study of radial extracorporeal shock wave therapy for spastic plantar flexor muscles in very young children with cerebral palsy. Medicine. 2016; 95(19):3649. doi: 10.1097/md.0000000000003649
[11] Li TY, Chang CY, Chou YC, Chen LC, Chu HY, Chiang SL, et al. Effect of radial shock wave therapy on spasticity of the upper limb in patients with chronic stroke. Medicine. 2016; 95(18):3544. doi: 10.1097/md.0000000000003544

[12] Daliri SS, Forogh B, Emami Razavi SZ, Ahadi T, Madjlesi $\mathrm{F}$, Ansari NN. A single blind, clinical trial to investigate the effects of a single session extracorporeal shock wave therapy on wrist flexor spasticity after stroke. Neuro Rehabilitation. 2015; 36(1):67-72. doi: 10.3233/NRE-141193

[13] Manganotti P, Amelio E. Long-term effect of shock wave therapy on upper limb hypertonia in patients affected by stroke. Stroke. 2005; 36(9):1967-71. doi: 10.1161/01. str.0000177880.06663.5c

[14] Sohn MK, Cho KH, Kim YJ, Hwang SL. Spasticity and electrophysiologic changes after extracorporeal shock wave therapy on gastrocnemius. Annals of Rehabilitation Medicine. 2011; 35(5):599. doi: 10.5535/arm.2011.35.5.599

[15] Moon SW, Kim JH, Jung MJ, Son S, Lee JH, Shin H, et al. The effect of extracorporeal shock wave therapy on lower limb spasticity in subacute stroke patients. Annals of Rehabilitation Medicine. 2013; 37(4):461. doi: 10.5535/ arm.2013.37.4.461

[16] Santamato A, Francesca Micello M, Panza F, Fortunato F, Logroscino G, Picelli A, et al. Extracorporeal shock wave therapy for the treatment of poststroke plantar-flexor muscles spasticity: A prospective open-label study. Topics in Stroke Rehabilitation. 2014; 21(1):17-24. doi: 10.1310/ tsr21s1-s17

[17] Marinelli L, Mori L, Solaro C, Uccelli A, Pelosin E, Currà A, et al. Effect of radial shock wave therapy on pain and muscle hypertonia: a double-blind study in patients with multiple sclerosis. Multiple Sclerosis Journal. 2015; 21(5):622-9. doi: $10.1177 / 1352458514549566$

[18] Lee JY, Kim SN, Lee IS, Jung H, Lee KS, Koh SE. Effects of extracorporeal shock wave therapy on spasticity in patients after brain injury: A meta-analysis. Journal of Physical Therapy Science. 2014; 26(10):1641-7. doi: 10.1589/jpts.26.1641

[19] Ghotbi N, Ansari NN, Naghdi S, Hasson S, Jamshidpour B, Amiri S. Inter-rater reliability of the Modified Ashworth Scale in assessing lower limb muscle spasticity. Brain Injury. 2009; 23(10):815-9. doi: 10.1080/02699050903200548

[20] Cho KH, Lee HJ, Lee WH. Reliability of rehabilitative ultrasound imaging for the medial gastrocnemius muscle in poststroke patients. Clinical Physiology and Functional Imaging. 2013; 34(1):26-31. doi: 10.1111/cpf.12060

[21] Podsiadlo D, Richardson S. The timed "Up \& Go": A test of basic functional mobility for frail elderly persons. Journal of the American Geriatrics Society. 1991; 39(2):142-8. doi: 10.1111/j.1532-5415.1991.tb01616.x

[22] Ng SS, Hui-Chan CW. The timed Up \& Go test: Its reliability and association with lower-limb impairments and locomotor capacities in people with chronic stroke. Archives of Physical Medicine and Rehabilitation. 2005; 86(8):1641-7. doi: 10.1016/j.apmr.2005.01.011

[23] Sawant A. Measurement of joint motion: A guide to goniometry. Toronto: University of Toronto Press; 2004. doi: $10.2310 / 6640.2004 .00031$ 
[24] Sköld C. Spasticity in spinal cord injury: Self- and clinically rated intrinsic fluctuations and intervention-induced changes. Archives of Physical Medicine and Rehabilitation. 2000; 81(2):144-9. doi: 10.1016/s0003-9993(00)90132-1

[25] Kottner J, Audige L, Brorson S, Donner A, Gajewski BJ, Hróbjartsson A, et al. Guidelines for Reporting Reliability and Agreement Studies (GRRAS) were proposed. International Journal of Nursing Studies. 2011; 48(6):661-71. doi: 10.1016/j.jinurstu.2011.01.016

[26] Feinstein AR. Clinimetrics. New Haven: Yale University Press; 1987.

[27] Weir JP. Quantifying test-retest reliability using the intraclass correlation coefficient and the SEM. Journal of Strength and Conditioning Research. 2005; 19(1):231-40. doi: 10.1519/00124278-200502000-00038

[28] De Boer MD, Seynnes OR, di Prampero PE, Pišot R, Mekjavić IB, Biolo G, et al. Effect of 5 weeks horizontal bed rest on human muscle thickness and architecture of weight bearing and non-weight bearing muscles. European Journal of Applied Physiology. 2008; 104(2):401-7. doi: 10.1007/ s00421-008-0703-0

[29] Desrosiers J, Bourbonnais D, Bravo G, Roy PM, Guay M. Performance of the 'unaffected'upper extremity of elderly stroke patients. Stroke. 1996; 27(9):1564-70. doi: 10.1161/01. str.27.9.1564

[30] Yang YB, Zhang J, Leng ZP, Chen X, Song WQ. Evaluation of spasticity after stroke by using ultrasound to measure the muscle architecture parameters: A clinical study. International Journal of Clinical and Experimental Medicine. 2014; 7(9):2712-7. PMCID: PMC4211779 
